\title{
Characterization of intertidal flat hydrodynamics
}

\author{
P. Le Hir ${ }^{\mathrm{a}, *}$ W. Roberts ${ }^{\mathrm{b}}$, O. Cazaillet ${ }^{\mathrm{c}}$, M. Christie ${ }^{\mathrm{d}}$, \\ P. Bassoullet ${ }^{\mathrm{a}}$, C. Bacher ${ }^{\mathrm{e}}$ \\ a IFREMER, Centre de Brest, DEL/EC-TP, BP 70, 29280 Plouzané, France \\ ${ }^{\mathrm{b}}$ HR Wallingford, Howbery Park, Wallingford, Oxfordshire, OX10 8BA, UK \\ ${ }^{\mathrm{c}}$ SOGREAH Ingéniérie, B.P.172, 38042 Grenoble Cedex 9, France \\ ${ }^{\mathrm{d}}$ Institute of Marine Studies, University of Plymouth, Plymouth, PL4 8AA, UK \\ ${ }^{\mathrm{e}}$ CREMA, B.P. 5, 17137 L'Houmeau, France
}

Received 28 May 1999; received in revised form 27 January 2000; accepted 28 January 2000

\begin{abstract}
The paper reviews the different physical forcings that control tidal flat hydrodynamics. Tidal propagation and cross-shore or long-shore currents, tidal asymmetry, wind-induced circulation, wave propagation and drainage processes are successively considered. Some simple methods are described for estimating cross-shore currents and wave-induced bottom shear stresses, and the results obtained are compared to field measurements on three contrasted sites in Europe. In particular the cross-shore current is shown uniform in the lower part of the flat, and decreasing towards the shore. Bottom friction-induced wave attenuation is simply formulated on gently sloping beds, leading to a maximum wave height that a flat can experience; it is proportional to the water height according to the ratio between the slope and the wave friction factor. The maximum related shear stress occurs at high water and is also proportional to the water depth. Maximum tidal velocities are very similar in the three sites where bottom sediment is muddy, suggesting a relationship between physical stresses and sediment characteristics. The consequences of physical forcings on sediment transport are listed. The bottom shear stress is suggested as the relevant parameter for comparing tidal and wave effects. In general, tide induces onshore sediment transport, whereas waves and drainage favour offshore transport. The processes leading to a possible tidal equilibrium profile are analysed: they involve the intrinsic asymmetry that favours net deposition at high water, and an ebb dominance generated by the resulting bottom profile convexity. Eroding waves are likely to upset such a balance; this equilibrium then reduces to a trend for the system. (C) 2000 Elsevier Science Ltd. All rights reserved.
\end{abstract}

Keywords: Hydrodynamics; Tidal flats; Waves; Drainage; Tidal asymmetry; Energy dissipation; Bottom stress; Mudflat; Geomorphology

* Corresponding author. Tel.: + 33-298-22-43-40; fax: + 33-298-22-45-94.

E-mail address: plehir@ifremer.fr (P. Le Hir). 


\section{Introduction}

Tidal flats are characterized by rapidly varying water depths, both in time and space, with the additional air exposure. These strong water elevation fluctuations are likely to induce specific hydrodynamics, which in turn generate particular sediment processes. Hydrodynamics consist of both the flow and water depth time/space evolution, which are generally strongly correlated (onshore current when water elevation rises, offshore current when it falls). These forcings are responsible for advection and dispersion, but they also generate bottom shear stresses, which are relevant for sediment erosion and deposition. The bottom shear stress is the combined effect at the bed of non-linear interactions between the mean flow and the waves.

A review of physical processes and their effect on sediment transport in intertidal areas has been recently presented by Eisma and Ridderinkhof (in Eisma, 1997). The main forcings that a coastal system can experience are (1) the tide, (2) the windinduced circulation, (3) the waves, (4) the density-driven circulation and (5) the drainage process. Drainage processes are specific to intertidal areas when they are exposed and subjected to surficial water discharge.

Tide is naturally the most important process, as it determines the existence of the intertidal flat. Tidally induced currents can be split into a cross-shore component, which accounts for the filling and the emptying of the flat, and a long-shore component, which depends on the large-scale circulation around the flat.

Meteorological events are likely to generate large-scale variations of water surface elevation, and flow patterns that modulate and sometimes counteract the tidal currents.

Waves are either due to the propagation of offshore waves or generated by local winds. Even in sheltered areas, waves are seldom negligible, as small waves can be sufficient to resuspend soft cohesive sediments in very shallow areas, and often contribute to morphological stability in the long-term.

Many tidal flats are connected to estuarine systems where the salinity gradients induce a density-driven circulation that frequently dominates the tidal residual circulation. Another density effect occurs if there are strong temperature variations at the mudflat surface due to solar radiation (e.g. Guarini et al., 1997). These temperature variations may induce rapid changes in the local water temperature $\left(a 3^{\circ} \mathrm{C}\right.$ change in $1 \mathrm{~h}$ has been measured in a flat of the Humber estuary) and then thermal gradients may appear over the flat. However, the density gradients have baroclinic effects that become important in deep areas only. Thus over a tidal flat, the density-driven circulation can only develop around a connection with a neighbouring channel, and then it is mainly controlled by the large-scale circulation. For this reason, this forcing will not be considered in the present paper.

The last mentioned forcing is drainage. This encompasses both the flow of surficial freshwater from upstream, and the expulsion of porewater from the upper sediments as the water table lowers during falling tide. This latter process is favoured on muddy tidal flats where low permeabilities prevent the porewater to flow within the bed. Little is known about the effects of drainage processes upon tidal flat evolution, although observations indicate the importance of drainage effects for the sediment 
transport (e.g. Bassoullet et al., 2000). Drainage also appears an important factor for long-term stability, when drainage-modulated seawards transport compensates for a tidally induced residual accretion of the flat (Bessineton, pers. com.).

Assessing the contribution of these five forcing processes to the bottom stress is a difficult problem, not specific to tidal flats. However, for the intertidal zone, bed irregularities are more likely, because of drainage, drying and swelling events in muddy areas, bioturbation by benthic animals and protection by plants. In addition, the computation of shear stresses is complicated by extremely shallow water depths, for which classical laws are questionable.

The purpose of this paper is to characterize the water movements (waves and current) over intertidal areas, considering in turn tidal propagation and induced currents, wind-induced circulation, waves and drainage.

As shown by Friedrichs and Aubrey (1996), the hydrodynamics and morphodynamics are interrelated. This study focuses upon the dependence of the flows or waves on the topography, whilst the interactions between the hydrodynamics and sediment transport, leading to possible equilibrium profiles, are analysed in detail by Roberts et al. (2000).

\section{Investigated sites}

The work is mainly based on observations and computations on three sites which have been investigated within the INTRMUD project, funded by the European Community. By considering the consequences of hydrodynamics on sediment dynamics and morphological equilibrium, the paper contributes to the INTRMUD classification of intertidal flats (Dyer et al., 2000). The three investigated sites are (Fig. 1):

(1) the Brouage mudflat in the Marennes-Oléron bay, on the western coast of France; the intertidal area is $4 \mathrm{~km}$ wide, with a mean bottom slope of $1 / 800$, which is consistent with a tidal range of $5 \mathrm{~m}$; the area is sheltered from Atlantic swells by the Ile d'Oléron; the flat is entirely muddy, with ridges and runnels on the flatter section (slope $\approx 1 / 1400$ ) in the middle of the flat (Bassoullet et al., 2000).

(2) the Skeffling mudflat $10 \mathrm{~km}$ inside the mouth of Humber estuary (England), where it opens into the North Sea. Tidal range is $6 \mathrm{~m}$, flat width is $4 \mathrm{~km}$ and the mean slope is $1 / 700$, but in the order of $1 / 1300$ in the medium section of the flat. The sediment is predominantly muddy on the upper part, but becomes mixed and even sandy towards the lower levels. Intriguingly, this flat exhibits similar shore normal ridges and runnels in the middle of the profile (Whitehouse et al., 2000) as the Brouage mudflat.

(3) the Northern intertidal flat of the Seine estuary, near Le Havre (France), connected to the English Channel. The area is macrotidal (range $=7 \mathrm{~m}$ ) and the tide is characterized by strong asymmetries and a large duration of high water slack (about $3 \mathrm{~h}$ ). The mudflat slope (1/150) is larger than in the previous flats and the waves are stronger, as the site remains open to the extended Baie de Seine. 

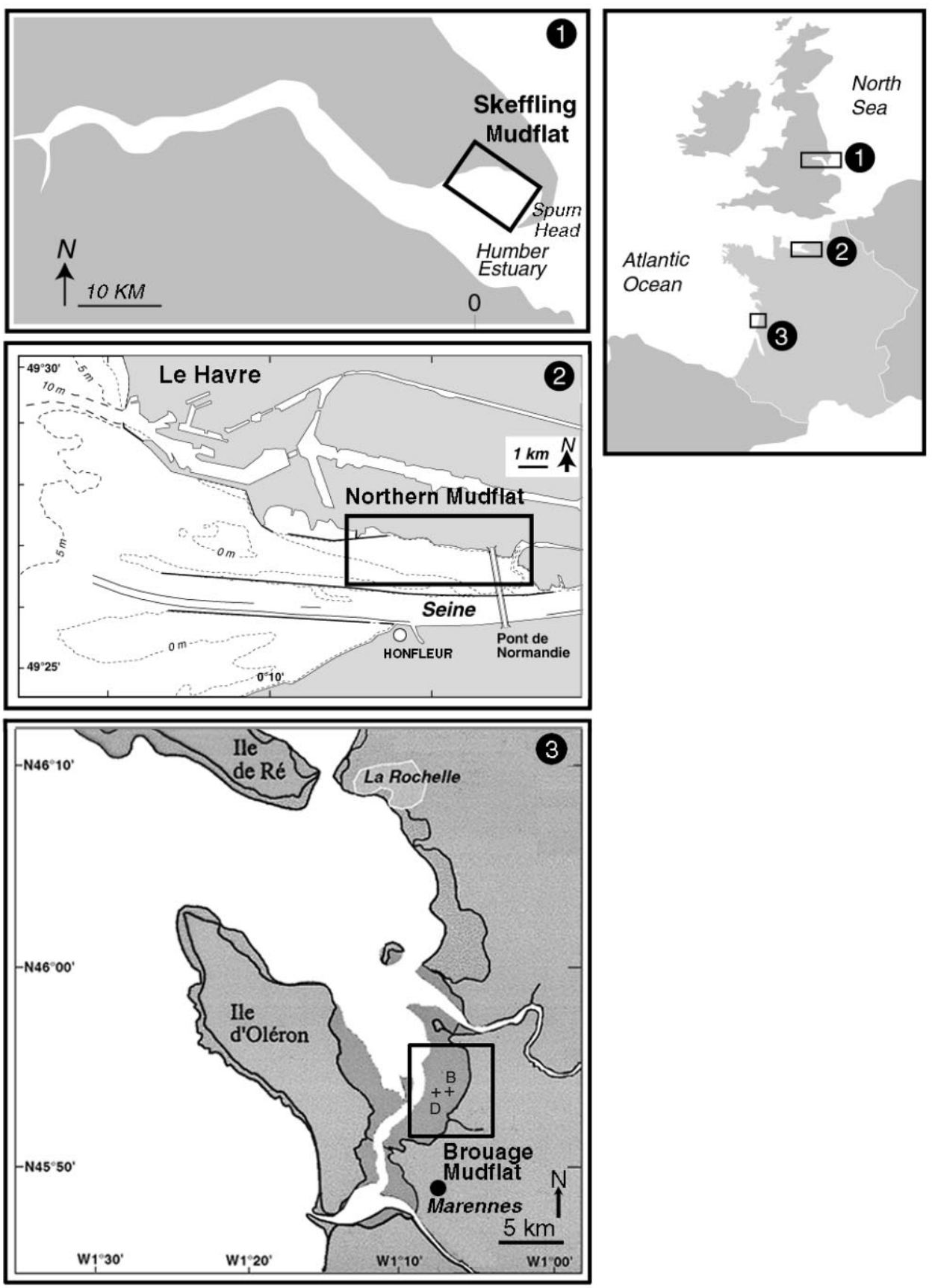

Fig. 1. Location of the 3 investigated sites: (1) Skeffling mudflat in the Humber estuary (UK); (2) Northern mudflat in the Seine estuary (Fr.); (3) Brouage mudflat in the Marennes-Oléron Bay (Fr.); $D$ and $B$ are the measurement stations. 
Sediment is sandy in the lower part and mixed or muddy everywhere else on the flat, but annual monitoring measured frequent changes in the sediment nature.

The following text gives an analysis of different hydrodynamic features, mostly based on data from these sites. These data can be records of flow velocity and pressure (giving the tide and waves) at fixed stations (e.g. Bassoullet et al., 2000; Christie et al., 1999), or results of depth-averaged modellings. The latter have been performed with the SAM-2DH model (Brenon and Le Hir, 1999) for the Marennes-Oléron bay, and the Télémac-2D model for the Humber estuary (Roberts and Whitehouse, 2000) and for the Seine estuary.

\section{Tidal flow}

\subsection{Tide propagation}

The wave length of the tide is much larger than the flat width so that the water elevation is nearly horizontal at the scale of the flat. This feature can be weakened by two factors: first the tidal wave which propagates at the celerity $(g h)^{0.5}$ can be retarded in very shallow waters (e.g. at the tidal front), but usually only for a very short period. Then the surface elevation may present a significant slope, due to a supercritical regime. Indeed, small bores are sometimes observed on tidal flats (e.g. in Dronkers, 1986), but these bores only affect the leading water's edge. Secondly, bottom friction can also slow down the tidal wave propagation, especially in shallow waters and across gentle slopes (e.g. Friedrichs and Madsen, 1992).

These effects have been considered for the cross-shore propagation of the tide. Even when the tide is mainly propagating along the shore, the phase lag between the shore and the deep area off the flat remains controlled by the cross-shore propagation. A one-dimensional model that solves the depth-averaged shallow water equations has been used. The convex bottom profile is the result of morphological computations (see Roberts et al., 2000) and is typical of tidally influenced mudflats (Friedrichs and Aubrey, 1996; Lee and Mehta, 1997). Fig. 2 shows the slight deviation of the surface elevation from the horizontal, and the currents associated to the tide propagation. Noticeable surface slopes only occur behind the tidal front. When accelerations are neglected, the resolution of water volume conservation between two successive horizontal water surfaces leads to depth-averaged currents very similar to the ones computed by the shallow water model. This validates the hypothesis of nearly instantaneous cross-shore tide propagation.

The flow field can be split into a cross-shore component and a long-shore one, the latter being more related to large-scale processes and generally stronger.

\subsection{Cross-shore tidal currents}

The magnitude of the cross-shore current depends mainly on the width of the mudflat. It can exceed the long-shore current when the intertidal flat is particularly 

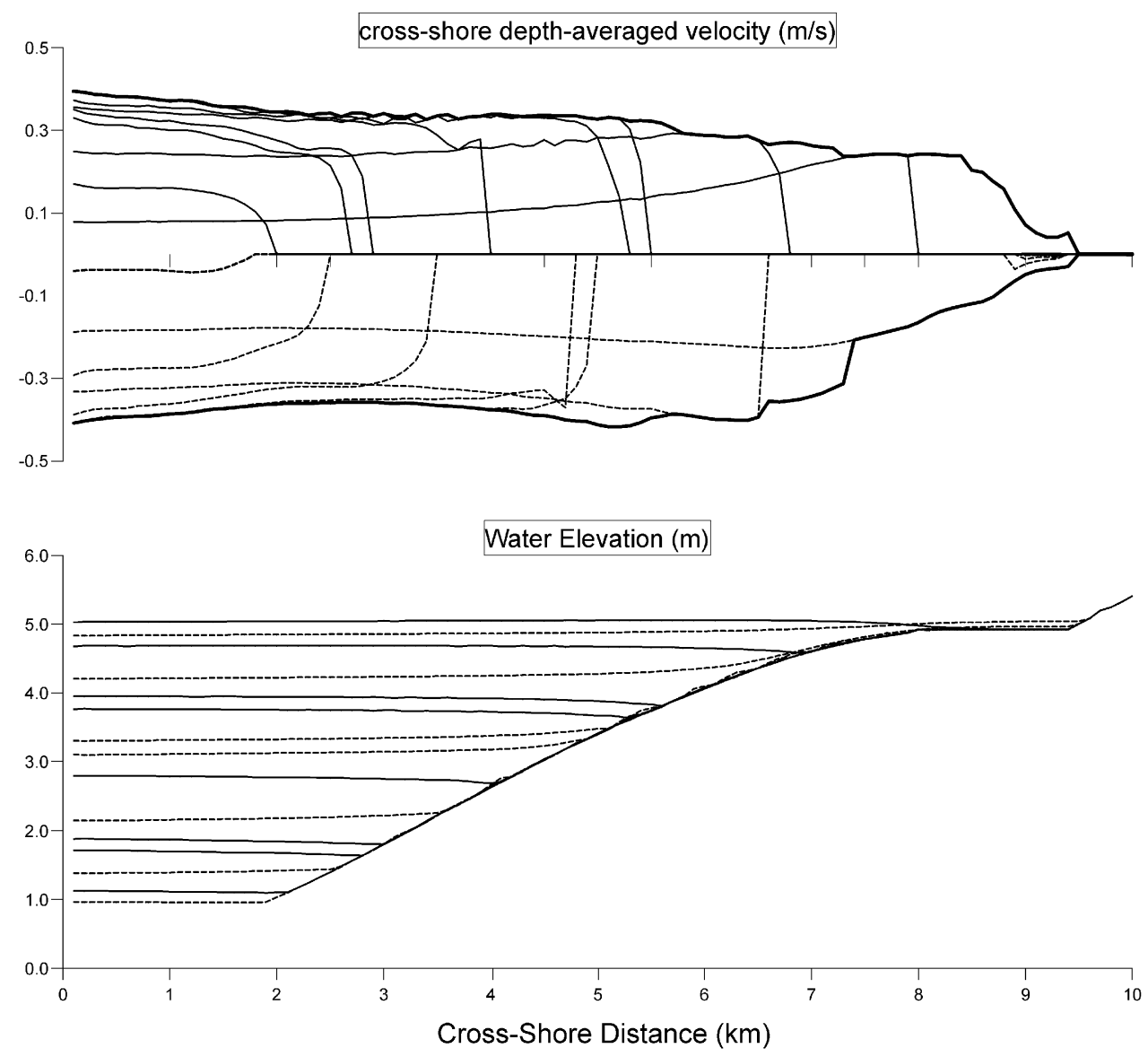

Fig. 2. Cross-shore simulation of tide propagation and induced currents over an intertidal flat. Dotted lines are ebb/falling episodes. Thick curves are envelopes of maximum flood or ebb velocities over the tide.

wide (e.g. the Brouage mudflat), and/or when the alongshore currents are reduced by the presence of a natural or man-made obstruction, such as a headland, spit or breakwater (e.g. the Skeffling flat, Fig. 3).

In the case of uniform bottom slope $\beta$, it can be shown (Appendix A; Friedrichs and Aubrey, 1996) that at any location of the flat below mean water level, the maximum cross-shore current is approximately

$$
u_{\max }=\frac{\pi R}{\beta T_{\text {tide }}}
$$

where $R$ and $T_{\text {tide }}$ are, respectively, the tidal range and period. In practice, the slope of many estuarine mudflats around the mean water level is flatter than the average slope 


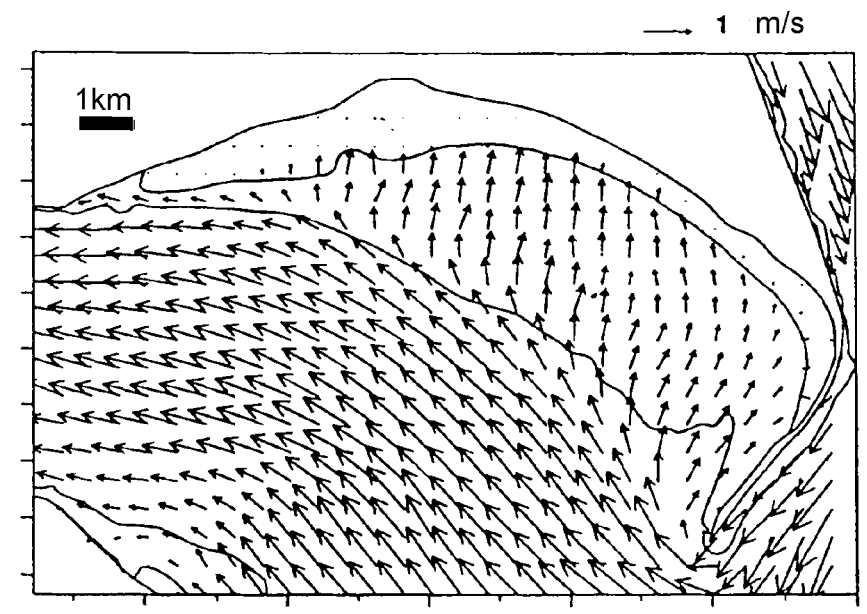

Fig. 3. Computed current field on the Skeffling flat (Humber estuary), 2h30' before high water. Bathymetry contours shown are LWS and MWL. (After Roberts and Whitehouse, 2000).

Table 1

\begin{tabular}{llll}
\hline Site & Tidal range $(\mathrm{m})$ & Bottom slope & $U_{\max }\left(\mathrm{m} \mathrm{s}^{-1}\right)$ \\
\hline Brouage & 5 & $1 / 800$ & 0.28 \\
flatter section & & $1 / 1400$ & 0.49 \\
Skeffling & 6 & $1 / 700$ & 0.29 \\
flatter section & & $1 / 1300$ & 0.55 \\
Le Havre & 7 & $1 / 150$ & 0.07 \\
\hline
\end{tabular}

from high-to-low water, leading to a greater peak current than that indicated by Eq. (1). For the three investigated sites, Eq. (1) gives the result of Table 1.

On the upper flat (above mean sea level), Friedrichs and Aubrey (1996) show that the maximum current occurs at the tidal front, and the intensity is given by

$$
u_{\max }=\frac{\pi R}{\beta T_{\text {tide }}} \sqrt{1-\left(\frac{2 \beta}{R} x_{\mathrm{f}}\right)^{2}}
$$

where $x_{\mathrm{f}}$ is the location of the tidal front (set equal to 0 at mid-tide).

For a linearly sloping flat, above the mean sea level, maximum cross-shore currents decay when approaching the shore.

These schematic results, which have been extended for more complicated geometries by using hypsometry data (Pethick, 1980; Friedrichs and Aubrey, 1996), can be compared to field observations or computations from depth-averaged models of the investigated flats. Fig. 4 gives representative examples of water level and current, recorded at station B on the Brouage flat (Fig. 4(a)) or computed on several locations 
(a)
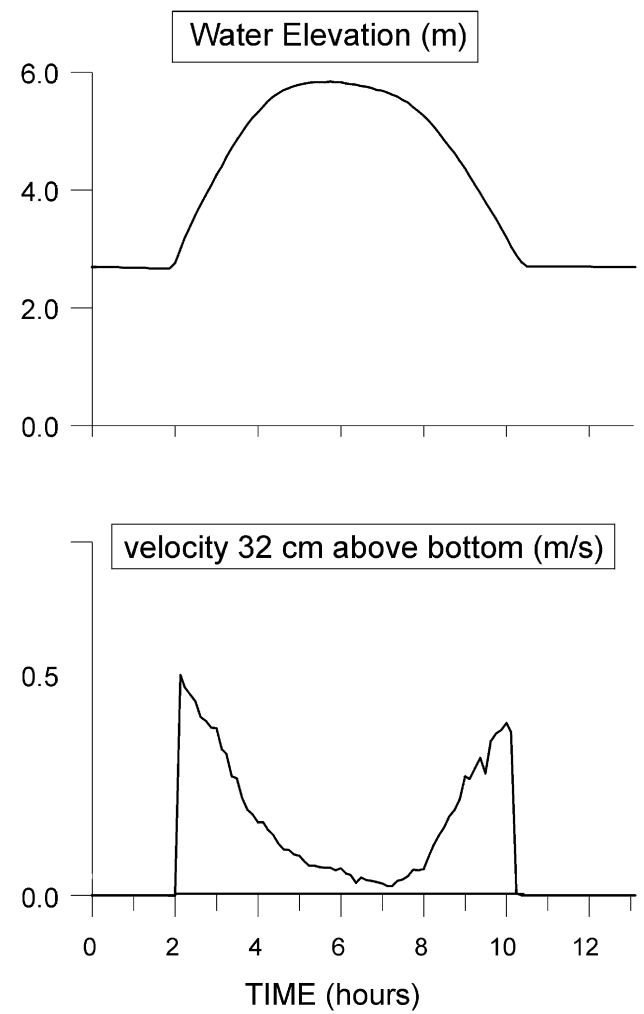

(b)
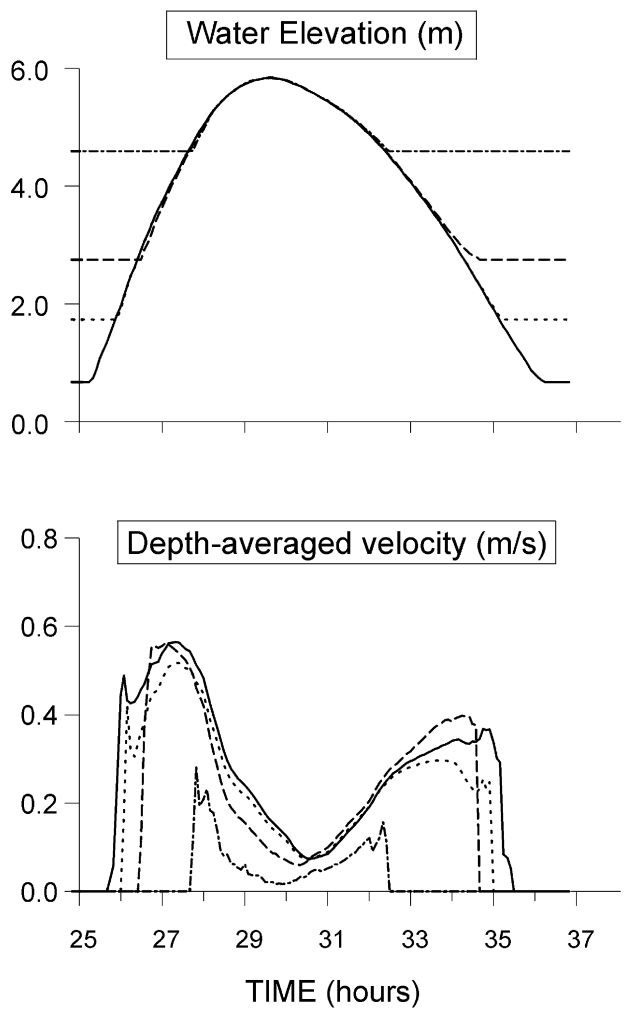

Fig. 4. Tide elevation and current velocities in the Brouage mudflat (Marennes-Oléron Bay, Fr): (a) measurements at station B, Nov.1994; (b) computations at 4 locations along a cross-section of the flat.

of the same flat (Fig. 4(b)). As stated before, the flow is mainly cross-shore at this site. According to the measurements as well as the model, maximum flood velocity occurs just after covering, approximately at mid-tide; after a long high water slack, the ebb progressively increases until a maximum speed just before uncovering. This corroborates the fact that maximum velocities are reached at mid-tide, or near the tidal front in the upper flat. The maximum velocity is about $0.45 \mathrm{~m} \mathrm{~s}^{-1}$, in agreement with the order of magnitude of a depth-averaged velocity deduced from Eq. (1). In addition, the model produces a relatively uniform maximum velocity in the lower part of the flat (up to mid-level) and a lower velocity in the upper flat, as indicated by relations (1) and (2).

Fig. 5 presents the computed maximum velocity (over a mean spring tide) at several locations for the three investigated sites versus the "reduced" maximum water depth. The latter is defined as the maximum water height over the tide divided by the tidal range and represents a normalized distance to the shore when the bottom slope is uniform. In contrast with the Humber and Brouage results, on the Seine site velocities 


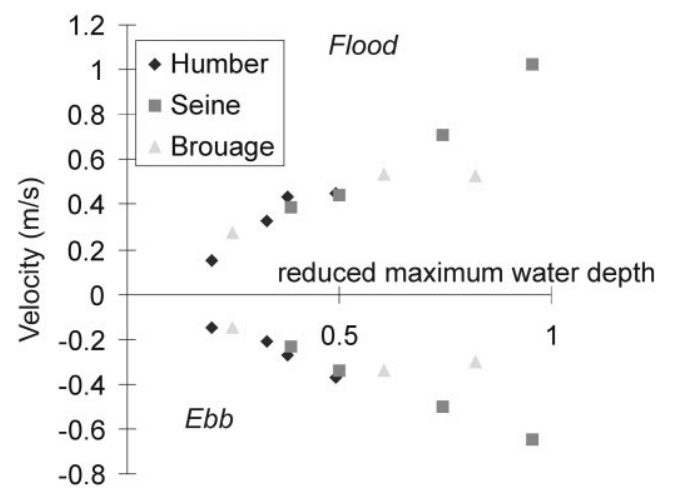

Fig. 5. Distribution of computed maximum velocities at different locations of the three investigated sites (mean spring tide). The "reduced maximum water depth" is the local maximum water height over the tide, divided by the tidal range.

increase with distance from the shore, even in the lower flat. In addition these velocities are considerably higher than the theorical cross-shore component deduced from Eq. (1). In fact, the flow is mainly longshore on this flat, and thus controlled by the large scale tidal propagation. However, in the upper part of the three sites, the total velocities are remarkably similar, which probably explains the analogy between the corresponding sediments. Actually, the lower part of the Seine flat is nearly sandy, where the tidal flow is larger. Lastly, the three sites exhibit a similar tidal asymmetry in favour of flood, which will be discussed below.

\subsection{Long-shore tidal currents}

Currents parallel to the shore are dependent on large scale topography. Typically, the volume of water which must pass through an estuary cross-section during a tidal cycle is determined by the tidal range and the shape of the estuary landward of that section. The distribution of flow across the section can be approximated by relating the current speed to the square root of the water depth. This arises from balancing the bed friction distributed over the water column $(\tau / h)$ and the longitudinal water surface slope in the depth-averaged momentum equation, assuming this slope is uniform across the section, which is reasonable when the flat is not too wide. More precisely, the bed friction is generally formulated as a quadratic function of the depth-averaged velocity, modulated by a function of the water height that represents the integration of velocity profile [for instance $1 / \operatorname{Ln}\left(h / e z_{0}\right)$ for a logarithmic profile]: as a result, the dependence of current speed on the water height across the flat can be slightly larger than the square root.

The Northern flat of the Seine estuary is representative of relatively narrow intertidal flats connected to a macrotidal estuary where currents are strong. Fig. 6 shows that simultaneous currents computed on different stations of the flat are actually correlated to the power $0.5+1 / 6$ of the local water depth, which is in 

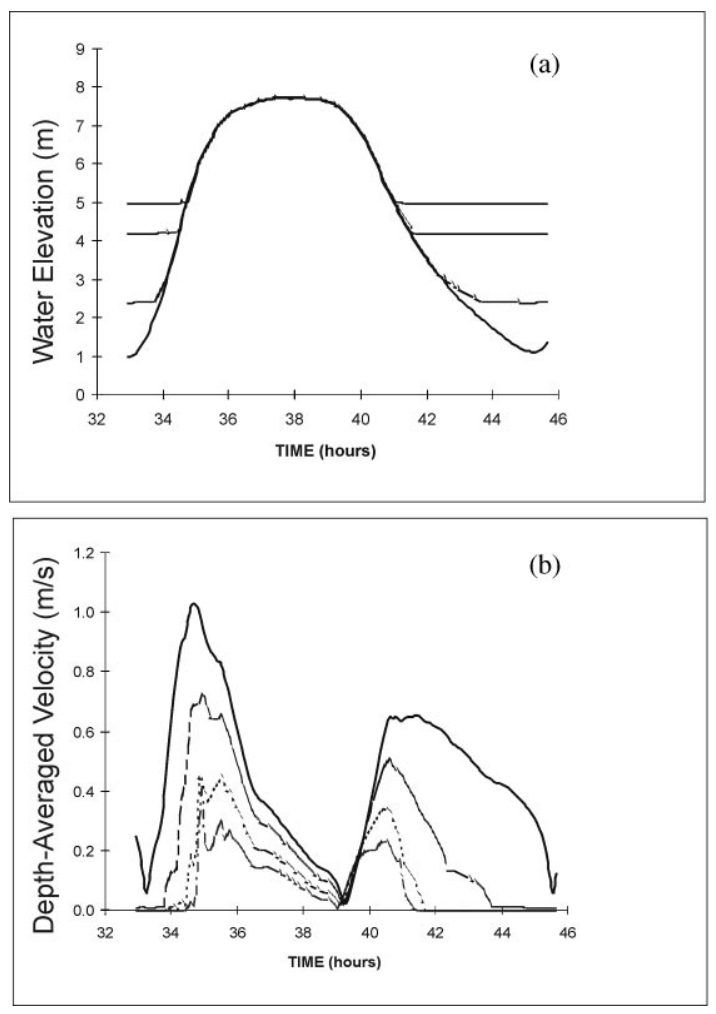

(c)

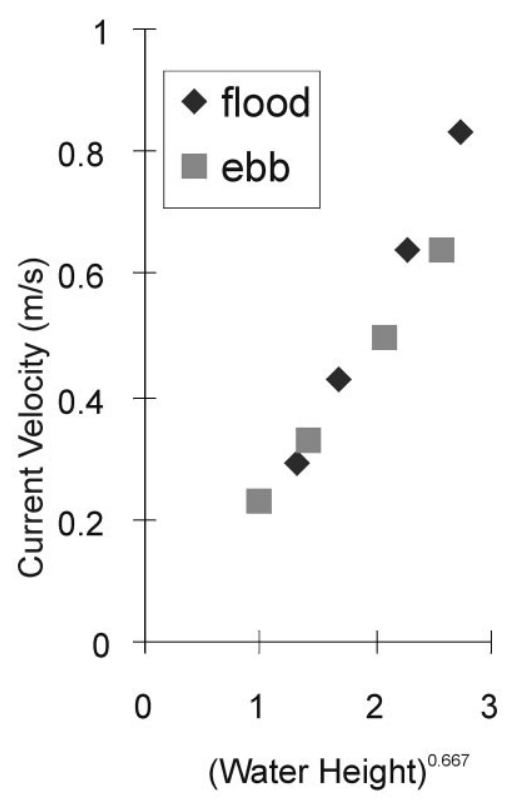

Fig. 6. Computed tidal characteristics along a cross-section of the Northern flat in the Seine estuary: (a) water elevation, with noticeable tide distortion; (b)depth-averaged velocity, with strong flood dominance; (c) correlation between velocity and water height, at typical instants of the tide.

agreement with the Stricker formulation of the bottom friction $\left(\tau \sim u^{2} / h^{1 / 3}\right)$ used in the model.

This kind of relationship can be modified to some extent by lateral diffusion, which tends to partially even out current speeds across the estuary, and by wind-induced currents. Also, at the time of peak current in the main channel, water depth on the mudflat is often very small, so that the lower intertidal flat is more strongly influenced by long-shore currents than the upper flat.

\subsection{Tidal asymmetry}

We can distinguish three kinds of tidal asymmetry which are likely to induce a net, tidally averaged sediment transport over a tidal flat:

(i) an intrinsic asymmetry due to the air exposure,

(ii) large-scale asymmetries due to tide propagation in the surrounding basin,

(iii) local asymmetries generated by the topography of the flat. 
Types (ii) and (iii) induce differences between the average magnitude of flood and ebb currents, whereas all kinds of asymmetries generate differences in the duration of the slack water period, type (i) being the more relevant in the case of intertidal flats. As pointed out by Eisma and Ridderinkhof (ibid) among others, the difference between flood and ebb magnitudes is more important for bedload transport, whereas the difference in slack durations is fundamental for suspended matter transport.

The intrinsic asymmetry is merely the fact that the upper part of an intertidal flat experiences only one slack at high water. As a consequence, particles coming onshore during the flood begin to settle onto the upper flat when the bottom stress is below its critical value for deposition. Deposition processes are favoured by the decreasing velocities near the shore. This allows time for the partial consolidation of newly deposited cohesive material. The subsequent resuspension of the deposited material requires the ebb flow speed to exceed some minimum, and often this minimum is hardly reached in the upper flat except if the ebb current is locally dominant. These processes, known as settling and scour lag effects, have been described by Postma (1961).

The large-scale asymmetry is caused by the non-linear distortion of the tide during propagation (advective and friction terms in the momentum equation), and is dependent on the geometry of the coastal area (e.g. Uncles, 1981). When the flat is connected to an estuary where the tide propagation is slowed down, the asymmetry is generally of flood dominant type. The tidal flow over the Northern flat in the Seine estuary (Fig. 6) exemplifies this kind of asymmetry. It should be noted that these large-scale asymmetries may vary, depending on the harmonic composition within for instance the fortnightly tidal cycle. This is illustrated in Fig. 7 which shows an inversion of the flow asymmetry on the Brouage mudflat, with a flood dominance at the beginning of the selected period and an ebb dominance at the end, for similar tidal ranges. Among tidal asymmetries, residual eddies due to headlands or islands have also to be mentioned, as they are likely to induce specific sediment transport or deposition (e.g. the Portishead mudflat, in the Severn estuary, Whitehouse and Mitchener, 1998).

The bottom topography of the flat can itself induce or modify the tidal asymmetry. Several authors pointed out that tidal flats with a "convex" profile enhance the maximum ebb curent (e.g. Dronkers, 1986). Actually the cross-shore computations plotted in Fig. 2 show an ebb dominance throughout the profile, except near the shore end.

To summarize, it can be said that large-scale topography induces asymmetries of the tide elevation and long-shore flow component, generally in the way of flood dominance, whereas local topography induces a cross-shore asymmetry.

\subsection{Bottom shear stresses and energy dissipation}

As stated in the introduction, bottom stress is the relevant parameter for assessing the deposition and erosion of sediments. It can be expressed as a quadratic function of the mean flow (or the flow at a given height), providing a drag coefficient (or equivalently a roughness length if a logarithmic velocity profile is assumed). This has been extensively analysed by Collins et al. (1998) who pointed out the strong 


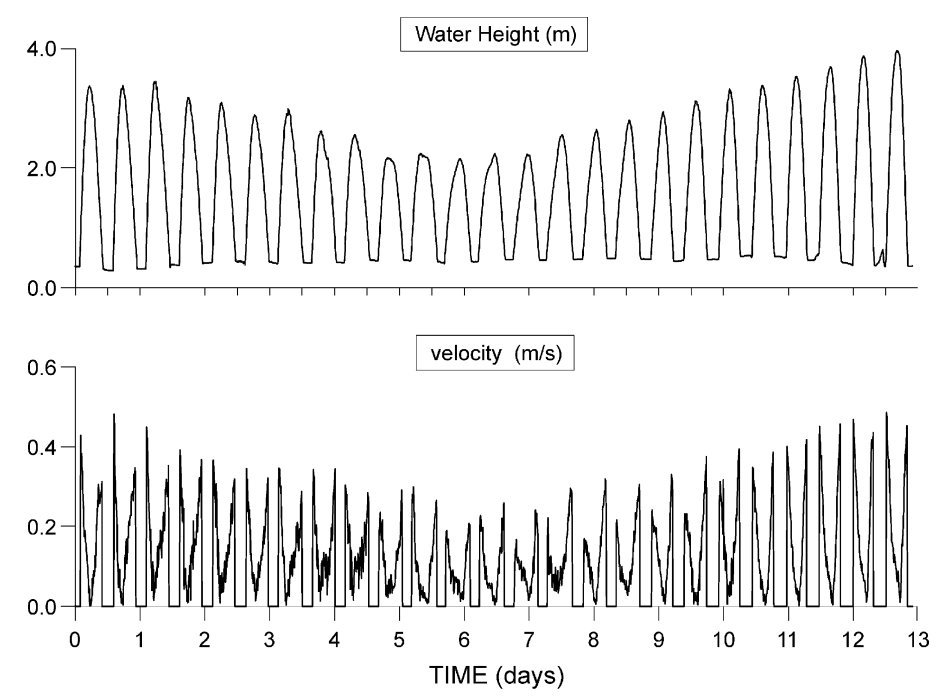

Fig. 7. Measured tide elevation and current velocity ( $32 \mathrm{~cm}$ above bottom) at station $\mathrm{B}$ in the Brouage mudflat over a fortnightly tidal cycle. Flood dominance appears from the beginning until the 4th day, whereas ebb is dominant between the days 6 and 12 .

variability of these parameters. Actually the determination of drag coefficients on intertidal areas is complicated by the presence of bedforms, ridge and runnel networks, channels, benthic fauna and plants. All these parameters can induce flow perturbations that modify the velocity profile and prevent representative bed shear stresses being calculated from local measurements. We thus prefer using the model results for characterizing the current-induced shear stresses at the scale of the flats.

For instance, Fig. 8 gives the distribution of the maximum bottom stress (over a spring tide) on the Skeffling mudflat. This shear stress was computed using a finite element depth-averaged model (TELEMAC-2D), assuming a uniform Nikuradse roughness of $0.01 \mathrm{~m}$. It can be seen that this tidal-induced peak shear stress is not uniform over the flat: it presents a strong decay towards the shore in the upper flat while it is more uniform in the lower half. These results are quite in agreement with the cross-shore velocity gradients predicted from Eqs. (1) and (2). As for the long-shore components, it should be reminded that they can be deduced from the hypothesis of uniform longitudinal surface slope across the flat: then from the depth-averaged momentum equation in steady regime, it is concluded that long-shore bottom stress components are proportional to the water depth and then also they decay shorewards.

Another commonly used parameter is the energy dissipation rate, assumed to be uniform when the flat is in equilibrium (e.g. Lee and Mehta, 1997). Computed as the work of the bottom stress, approximately proportional to the cube of the flow speed, it only enhances the contrasts presented by the bottom stresses. Finally, the distributions of shear stress and energy dissipation rate both reflect the velocity gradients and variations. The main advantage of these parameters over a simple velocity 

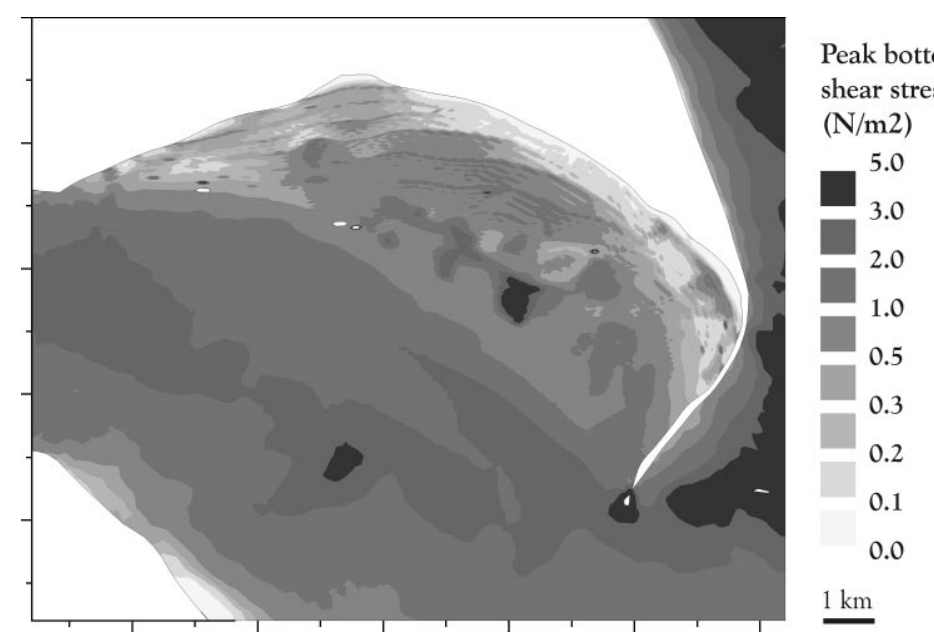

Fig. 8. Computed distribution of tidally induced shear stress over the Skeffling mudflat in the Humber estuary (maximum over a spring tide). (bottom roughness: Nikuradse coef. $=0.01 \mathrm{~m}$ ).

distribution is to enable a comparison with wave effects, and to be more relevant for the sediment behaviour.

\section{Wind-induced circulation}

Meteorological events act on water systems through atmospheric pressure gradients and wind. Pressure gradients can induce large-scale variations of water surface elevations. The time-scale of this process is generally too large for the related transient currents to be important, but the storm surge can significantly change the water depth on a flat (a few tens of $\mathrm{cm}$, or even more than $1 \mathrm{~m}$ ) and consequently change the air exposure as well as the local tidal flows. More frequently, winds are likely to generate a specific circulation by entrainment of surficial waters and generation of an additional surface slope, which in turn induces return flows in deeper waters.

In order to illustrate these wind effects, simulations of stormy winds over the Brouage mudflat have been performed, by using the depth-averaged model of the Marennes-Oléron Bay. A steady wind of $20 \mathrm{~m} \mathrm{~s}^{-1}$ was blowing eastward for $5 \mathrm{~h}$ either during a rising tide or a falling tide. Changes are noticeable on the upper flat (Fig. 9). Although the elevation is only enhanced by about $0.1 \mathrm{~m}$, the current speed is roughly doubled and asymmetry can reverse. Nevertheless at this location the bottom stresses remain small enough not to induce resuspension, so that the main consequence of the wind entrainment will be a modification in residual sediment transport and deposition.

Naturally, local winds generate waves that are likely to resuspend sediment, so that the correlation between the wind and wave episodes should be accounted for. 

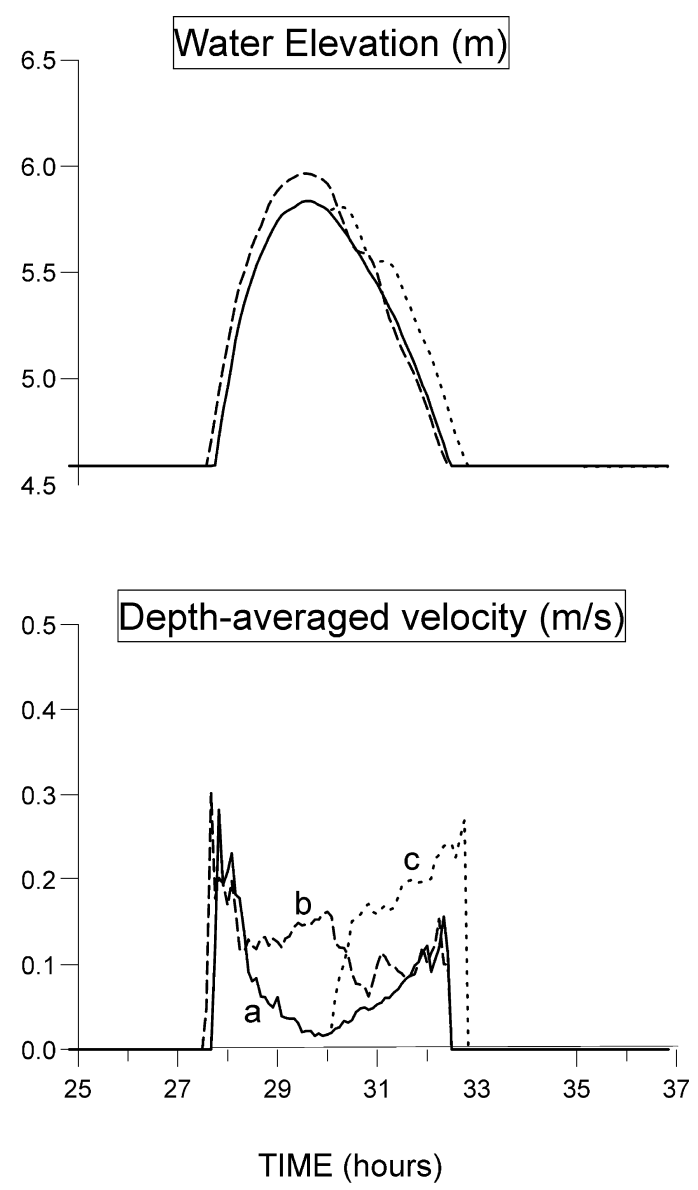

Fig. 9. Simulation of wind effects on hydrodynamics on the upper Brouage mudflat: (a) spring tide, without wind; (b) same tide, $20 \mathrm{~m} \mathrm{~s}^{-1}$ western wind blowing $5 \mathrm{~h}$ during the rising tide; (c) same tide, $20 \mathrm{~m} \mathrm{~s}^{-1}$ western wind blowing $5 \mathrm{~h}$ during the falling tide.

\section{Waves}

From one tidal flat to another, the wave regime can be very different: either the flat is exposed to offshore swells, or it is only submitted to short waves generated by local winds. In the first case, the flat is generally sandy and is nothing else than a beach. When nevertheless the area is muddy, because of a large input of sediment nearby, mud liquefaction is likely to occur and the waves experience strong damping (e.g. Wells and Kemp, 1986; Li and Mehta, 1997). In the second case, small waves can have an important influence on sediment transport, because, in shallow waters, the stress they cause on the bed is comparable with the stress associated with a quite substantial tidal current. 


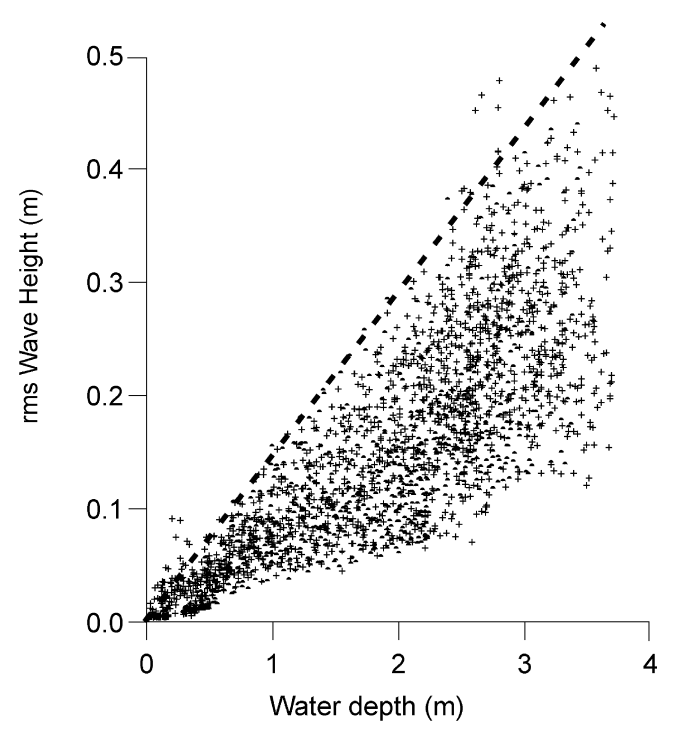

Fig. 10. Correlation between wave height and water depth measurements in the Brouage mudflat (France). Crosses: at station D, 17/26 Nov. 1997; dots: at station B, Oct-Nov. 1994; "rms wave height" is estimated as $2^{1.5}$ times the standard deviation of near bed pressure fluctuations. Dashed line: $H_{\mathrm{rms}}=0.15 \mathrm{~h}$.

\subsection{Wave height evolution on sheltered tidal flats}

The size of locally generated waves is dependent on the fetch length, the wind speed and the length of time for which the wind blows. For a fetch length of $5 \mathrm{~km}$ for example, the length of time for the waves to reach their fully developed state is around 30-40 mins, depending on the wind speed. In this respect, time is not usually a limiting factor, the wind speed, direction and fetch length are more important. For a given wind speed, wave height increases approximately as the square root of the fetch length. For a fixed fetch length, the wave height increases approximately linearly with wind speed. Then, when propagating over the flat, waves are likely to increase in height due to shoaling and fetch extension, and to decrease, due either to bottom friction or to visco-elastic entrainment of the bed.

Fig. 10 presents an overview of "root mean square" wave heights measured on two locations of the Brouage mudflat, during 20 days at station B on October 1994, and during 9 days at station $\mathrm{D}$ in November 1997. The root mean square wave height $H_{\text {rms }}$ is computed as $2^{3 / 2}$ times the standard deviation of the pressure fluctuations near the bottom, neglecting the wave pressure decay over the water column. It is striking that in both cases the plot exhibits a linear relationship between the maximum wave height and the local water depth, with the same slope at two different locations of the flat. Whatever the depth, the significant wave height does not exceed $15 \%$ of this depth. This can be compared to the ratio of 0.19 between $H_{\mathrm{rms}}$ and the water depth observed on the Surinam coast by Wells and Kemp (1986). They observed that the waves never broke because their height remained below the usual breaking limit 
$(H / h \sim 0.8)$, and they attributed the severe wave attenuation to dissipation into the bottom by the generation of a mudwave. Fluid muds are frequently observed near the sea bed along the Surinam, and may correspond to the case of exposed coasts previously mentioned. However, in the case of the Brouage mudflat, the liquefaction of the bottom does not seem to occur, at least under small or medium waves.

The reason for this "saturation" of the local wave height on flats can be looked for in bottom friction. Following the linear theory and applying the conservation of energy for a monochromatic wave propagating on a uniform slope with quadratic bottom friction, a relation can be found (see Appendix B) between the wave height $H$ and the distance from the shore $x$ according to the differential equation

$$
\frac{\mathrm{d}}{\mathrm{d} x}\left(H^{2} x^{1 / 2}\right)=\frac{2}{3 \pi} \frac{f_{\mathrm{w}}}{\beta^{2}} H^{3} x^{-3 / 2},
$$

where $f_{\mathrm{w}}$ is the wave friction factor.

This equation can be conveniently non-dimensionalized according to

$$
\frac{\mathrm{d}}{\mathrm{d} x}\left(H_{0}^{2} X^{1 / 2}\right)=\frac{2}{3 \pi} \frac{f_{\mathrm{w}}}{\beta} H_{0}^{3} X^{-3 / 2},
$$

where $H_{0}=H / h_{0}$ and $X=x \beta / h_{0}$, with $h_{0}$ the water depth at the offshore limit of investigation. $h_{0}$ should not be too large (typically $5 \mathrm{~m}$ ) because of the shallow water approximation.

Eq. (4) has been numerically integrated, and the solution is dependent on the relevant non-dimensional parameter $f_{\mathrm{w}} / \beta$ (Fig. 11). It can be seen that the trend of the wave attenuation is very different according to the value of this parameter. For low values of $f_{\mathrm{w}} / \beta$ (small friction or large bottom slope), dissipation is negligible and wave height is maintained or even increased (shoaling) until breaking, defined here as a maximum $H / h$ classically equal to 0.8 . On the other hand, for large values of $f_{\mathrm{w}} / \beta$ (high friction or gentle slope), dissipation becomes dominant and the wave height tends to a constant proportion of the water depth, whatever be the incident wave height. Then this proportion gives the maximum wave height such a tidal flat can experience at a given water depth. Assuming this proportion $(H / h)_{\text {lim }}$ exists, it can be computed from Eq. (3) by replacing $H$ by $h(H / h)_{\text {lim }}$ or $\beta x(H / h)_{\text {lim }}$, which after derivation leads to

$$
\left(\frac{H}{h}\right)_{\lim }=\frac{15 \pi}{4} \frac{\beta}{f_{\mathrm{w}}} .
$$

We assume that Eqs. (3) and (5) can be used with $H_{\text {rms }}$ instead of $H$, although the constant resulting from the integration of the dissipation rate could change according to the wave height distribution. Then the application of relation (5) for the case of the Brouage mudflat $\left(H_{\mathrm{rms}} / h\right)_{\mathrm{lim}}=0.15$ implies that the wave friction factor should be approximately 0.05 .

Measurements are also available in the upper section of the Northern mudflat in the Seine estuary. Waves and water depths have been recorded for 2 days during a storm (Fig. 12; Silva Jacinto et al., 1998). These measurements also show a linear relationship 
(a)

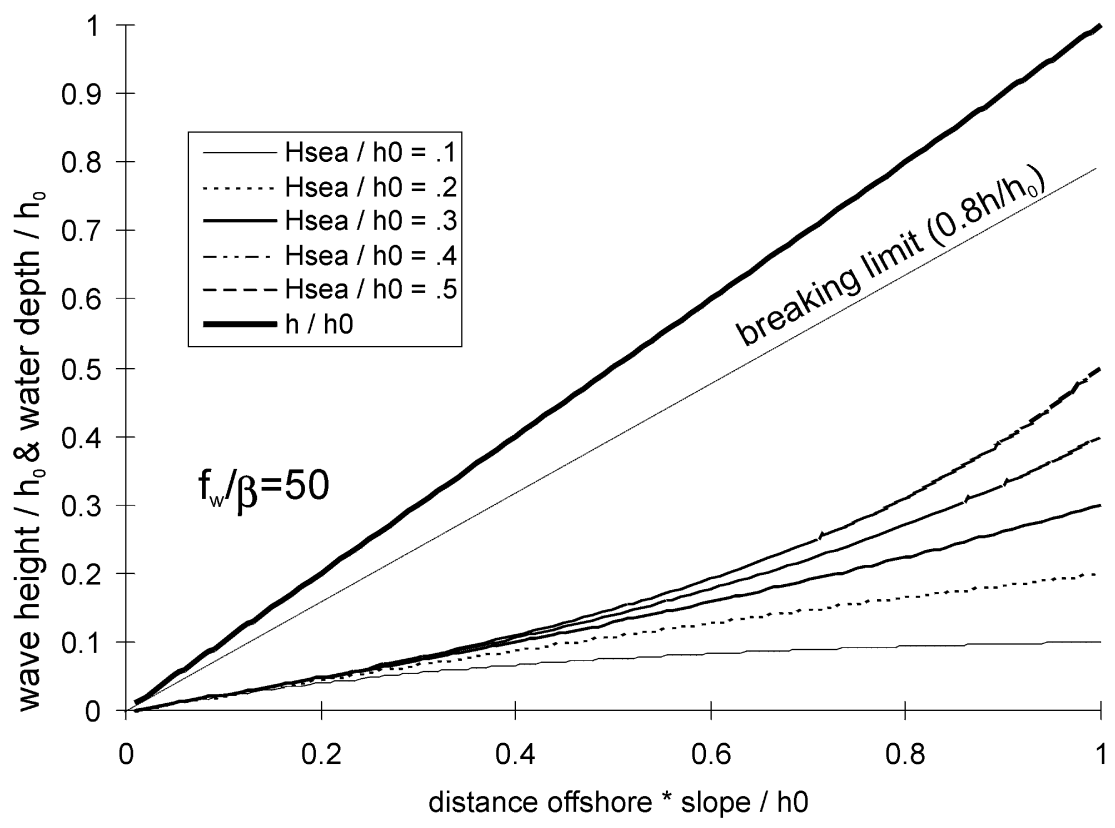

(b)

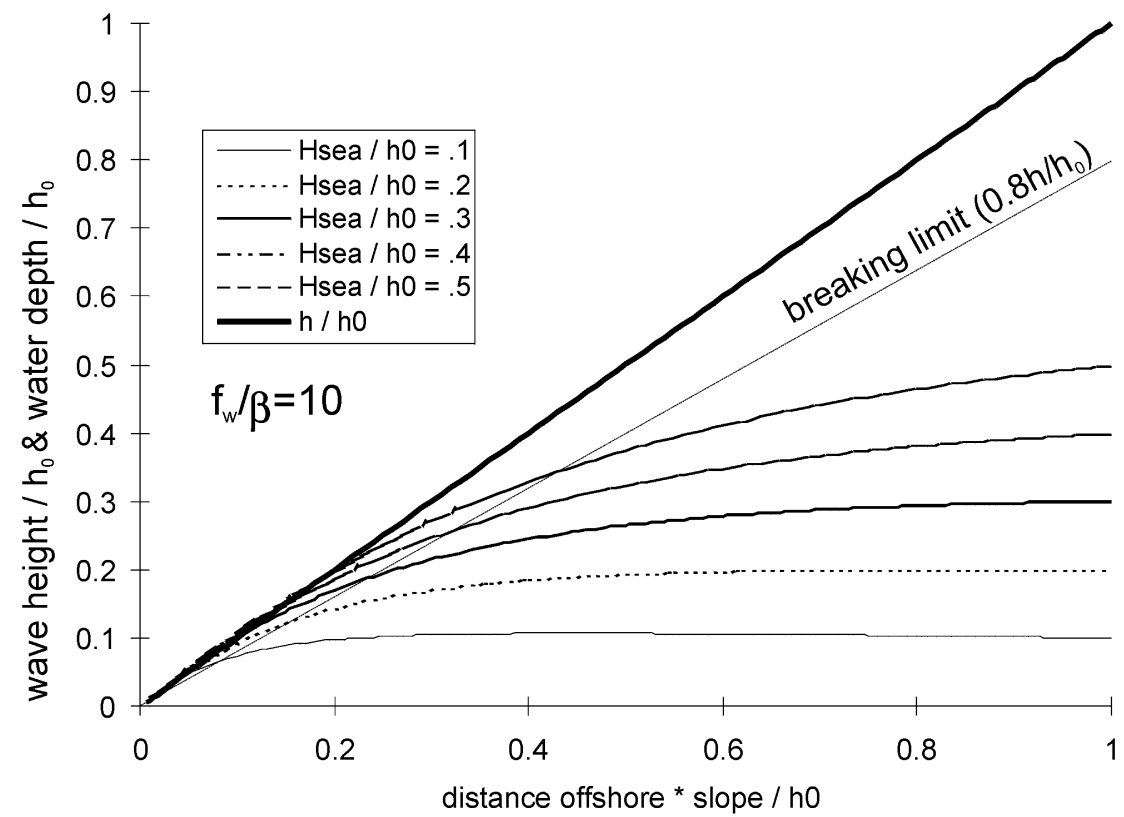

Fig. 11. Computed wave propagation over a linearly sloping bed (Eq. (4)) for different incident wave heights. The line " $h / h_{0}$ " represents the non-dimensionalized water depth. (a) $f_{\mathrm{w}} / \beta=50$ (wave friction factor $=0.05$, slope $=1 / 1000)\left(\right.$ b) $f_{\mathrm{w}} / \beta=10$ (wave friction factor $=0.05$, slope $=5 / 1000$ ). 


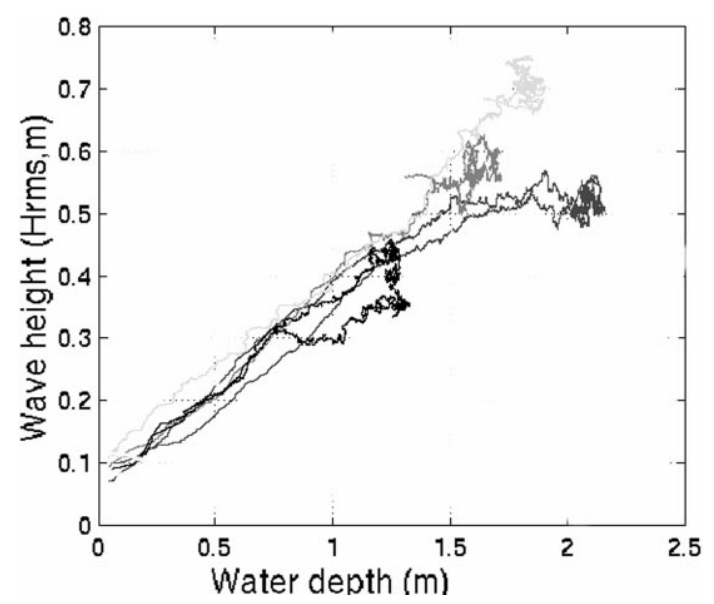

Fig. 12. Correlation between root mean square wave height and water depth, measured in the Northern mudflat of the Seine estuary (France), during a storm (12-14 feb.1997). (after Silva Jacinto et al., 1998).

between $H_{\text {rms }}$ and $h$, but the ratio is 0.28 , which is in agreement with the larger bottom slope of this flat. According to (5), the mean wave friction factor should be 0.14 , which is close to the upper limit of the usual range (from 0.005 to 0.4, Soulsby et al., 1993).

If the seabed consists of soft mud, then a significant proportion of the wave energy may be absorbed as it progresses across the bed. Lee and Mehta (1997) have addressed this case and discussed mechanisms of sediment transport by waves over a soft muddy bed, in the case of small tidal range.

\subsection{Wave-induced bed shear stress}

Combined with the shallow water approximation of the orbital velocity, the expression of the wave-induced shear stress (Eq. (3), Appendix B) becomes

$$
\tau=\frac{\rho g f_{\mathrm{w}}}{8} \frac{H^{2}}{h}
$$

Assuming a linear relationship between the maximum wave height and local depth, the bed shear stress reads

$$
\tau=\frac{\rho g f_{\mathrm{w}}}{8} \frac{\left[\min \left(H_{\mathrm{i}},(H / h)_{\mathrm{lim}} h\right)\right]^{2}}{h} .
$$

where $H_{\mathrm{i}}$ is the incident wave height. Shoreward of the zone where the maximum wave height to depth ratio is achieved, the shear stress decreases linearly with depth, as the wave moves towards the shoreline in the depth-limited zone. Seaward of the same transition zone, the stress variation is inversely proportional to the depth. This is 


\section{Water Elevation $(\mathrm{m})$}
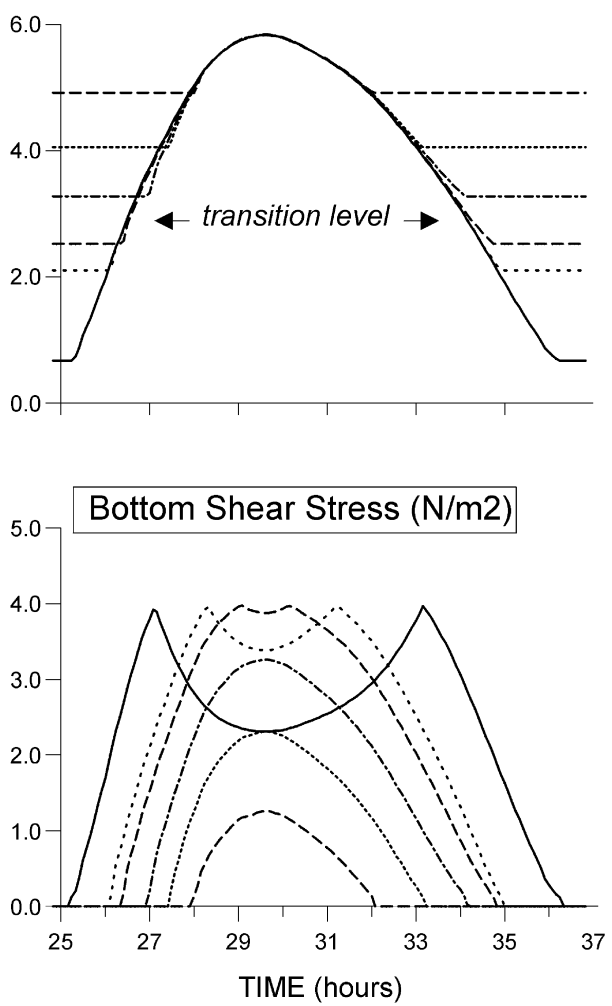

Water Elevation (m)
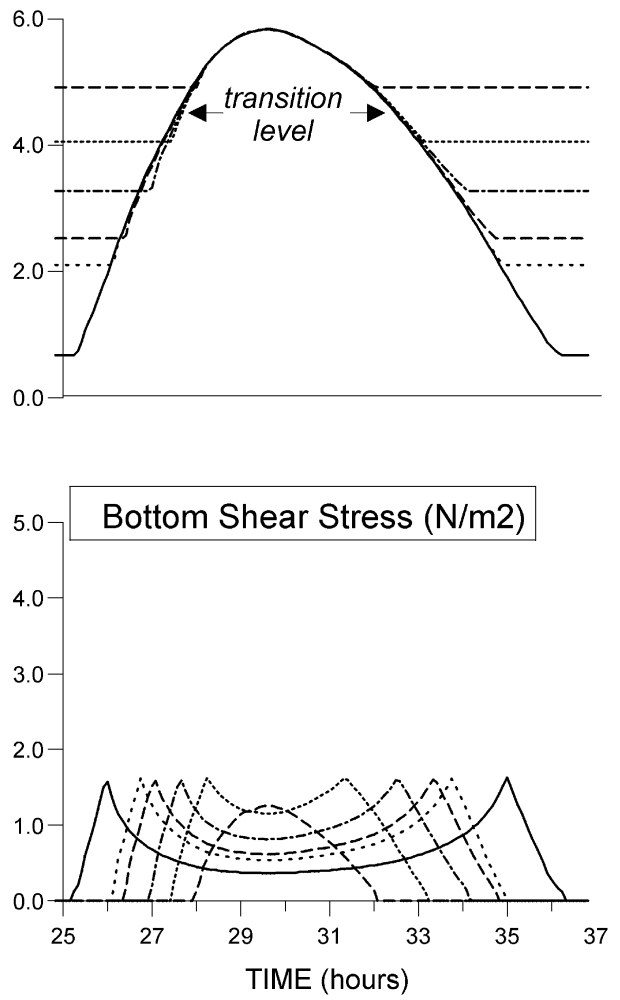

Fig. 13. Wave-induced bottom shear stress computed on different locations of the Brouage mudflat (Marennes-Oléron Bay, France), according to Eq. (6). Time evolution over a spring tide. (a) (left): incident wave height $=0.5 \mathrm{~m}$; (b) (right): incident wave height $=0.2 \mathrm{~m}$.

The "transition level" separates the upper fraction of the flat where the "saturation" wave height is reached (whatever be the incident wave) and the lower part where the maximum stress is uniform and depends on the incident wave.

shown in Fig. 13, which plots Eq. (6) at different locations of the Brouage mudflat during a spring tide, taking $(H / h)_{\lim }=0.15$. It should be noted that:

- the transition between the two trends is dependent on the value of the incident wave height $H_{0}$, for a given bottom slope;

- the upper flat experiences the highest stresses at high tide, whatever be the incident waves;

- the middle flat experiences the highest stresses a little after covering or before uncovering, when the water depth is $H_{\mathrm{i}} /(H / h)_{\text {lim }}$; for a given incident wave, this maximum stress is uniform;

- for a given bottom slope, the maximum stress that any location can experience is reached at high water when $H_{\mathrm{i}}=(H / h)_{\mathrm{lim}} h_{\mathrm{HW}}$, then it linearly increases with the water depth at high water $h_{\mathrm{HW}}$; 
- an increase of the bottom slope will delay or suppress the linear relationship between the stress and the water depth.

The order of magnitude of wind-induced stresses is at least the same as the one related to tidal flows. A major difference comes from that the former may be strong at high water, even in the upper flat, when the latter vanish. The consequence on sediment transport will be analysed in the discussion.

\section{Drainage}

Although drainage processes are commonly observed features on exposed tidal flats, very few studies have investigated this type of process. Drainage can be either due to creeks flowing from the shore at the surface of the flat (e.g. Whitehouse et al., 2000), or due to dewatering within the air-exposed sediment, when the water table is falling with some delay after the water surface (e.g. Anderson and Howell, 1984).

The water table gradient is supposed to generate pore-water flow inside the sediment, but in muddy environments, the low permeability forces the pore water to escape through the interface. When the bottom slope is rather steep (in the order of $1 \%$ or more), the water flows rapidly and evenly on the mud. When the slope is gentle, surficial flow is slow and a criss-crossed pattern of runnels which intersect each other is formed. These runnels grow and become organized progressively while running seaward, then forming a network of parallel ridges and runnels, cross-shore orientated. These patterns can be observed in the Brouage mudflat as well as the Skeffling mudflat (e.g. Whitehouse et al., 2000). The formation of the runnels by dewateringinduced surficial water flows is not clearly established, but the contribution of these longitudinal bedforms to drainage is undoubted, despite the fact that they retain water in the runnels at the end of the exposure period. As when the bottom slope is gentle cross-shore currents are dominant, the runnels are orientated along the tidal currents (and most often along the dominant waves, due to wave refraction). It seems that the tidal current enhances the organisation of runnels in a parallel network, contrasting with the meandering system which first occurs, without surprise over a flat bathymetry. The fact that ridge and runnel patterns extend in the section of the flat where the slope is minimum, i.e. where currents and bottom shear stresses are stronger (Eq. (1)), is noteworthy.

To the authors' knowledge, there has been no comprehensive investigation on the quantification of drainage-induced water discharge. If dewatering of the upper section of the flat is the only source, the discharge can be evaluated from the decrease of pore-water content of the mud, or even from the decline in sediment elevation. Assuming a mean reduction of $\delta h=1 \mathrm{~cm}$ for the sediment level during half of the mean air-exposure time, i.e. about $\delta t=10^{4} \mathrm{~s}$, the local sink of pore water corresponding to the sediment variation is $Q_{\mathrm{s}}=\delta h=0.01 \mathrm{~m}^{3} \mathrm{~m}^{-2}$. After a cross-shore distance $d=2000 \mathrm{~m}$ from the shore, the induced discharge can be evaluated as $Q_{\mathrm{d}}=Q_{\mathrm{s}} \cdot d / \delta t=0.002 \mathrm{~m}^{2} \mathrm{~s}^{-1}$. This means that to evacuate the pore water as surficial flow, the latter should be in the order of $0.002 \mathrm{~m}^{2} \mathrm{~s}^{-1}$ per unit width. If this flow is 
concentrated in runnels, with an active cross-section $A=0.2 \mathrm{~m} \times 0.1 \mathrm{~m}$ (where the water flows), every $2 \mathrm{~m}$ (the distance $\lambda$ between runnels), the current needed in runnels for discharging pore water is $U_{\mathrm{d}}=\lambda Q_{\mathrm{d}} / A=0.2 \mathrm{~m} \mathrm{~s}^{-1}$. This is actually the order of magnitude of velocities that are observed in the runnels. They are not negligible, and likely to transport suspensions and to prevent consolidation in the runnels.

Naturally runnels are not the only way of drainage. Channels are quite common features and contribute to drainage. They often coexist with runnels, and both systems may exhibit a slight angle between them, runnels being mainly parallel to the tidal currents and channels converging to the bathymetric gradient (Bassoullet et al., 2000).

\section{Discussion}

Most of the previous investigations result from simplifications of real cases, where generally all processes are combined. Mathematical models are the only tool which enables us to account for all these processes with a realistic topography. They are presently usable, at least for hydrodynamics, and some applications have been shown here. But our purpose in this paper is to point out the main features that we can simply deduce from the known characteristics of a given intertidal flat, without running a model which will take a long time to implement. This justifies the approximations for the tide propagation, and the choice of the simplest model for the waves.

However, not all the processes have been considered. For instance one can wonder how important is the rain, either as an additional source of water for drainage, or as a mechanism for roughness changes (e.g. Anderson, 1981). Similarly, fauna-induced bioturbation can change the roughness. The abundance of algae and plants is probably more essential, as they can slow down the water flows over the flat, and then favour sediment deposition (trapping). These "natural" processes and their consequences have been reviewed by Anderson (1981). One important feature is the seasonal variability that these biotic components can induce, and its consequence on sediment transport is becoming a topical subject that is beyond the topic of this paper.

Man may also modify the tidal flat hydrodynamics. For instance, shellfish farming installations, frequently set on the lower flats, are likely to change the current orientation and to dampen waves. This appears to be the case in the MarennesOléron Bay, and partly explains the wave attenuation observed on the Brouage mudflat. Little is known about the quantification of these processes. Boat waves constitute another forcing which is not very different from wind-induced waves, except that it can occur in sheltered areas close to channels, as for instance in upper estuaries.

\subsection{Tidal forcing and sediment response}

Back to the tidal forcing, we can consider the consequences on sediment processes of the different kinds of asymmetry that have been pointed out:

- the intrinsic asymmetry, which results from a single tidal slack between flood and ebb experienced by the upper flat, is responsible for a net sediment transport 
towards the shore, providing material is available (from the lower flat or from offshore); scour-lag effects (Postma, 1961) induce a net deposition on the upper flat, which favours the convexity of the bottom profile;

- the shape of the cross-shore profile can generate tidal current asymmetries; in particular, a tide-related convex profile enhances the maximum ebb current; the ebb current enhancement favours the seawards export of sediment, in contrast with the shorewards transport induced by the intrinsic asymmetry; this can lead to some sedimentological equilibrium, as indicated by morphological mathematical models under development (e.g. Roberts et al., 2000); for example, the convex bottom profile shown in Fig. 2, for which tidal currents exhibit an ebb dominance, results from the evolution of an initially linear profile over a period of 17 years, simulated by a cross-shore sediment transport model;

- large scale asymmetries are often related to the tide propagation in an estuary to which the flat is connected, and generally are flood dominant; at equilibrium, one can wonder whether this flood dominance should not be compensated by an increase of cross-shore convexity that would reinforce the ebb.

It can be noted that the tide-generated convexity compensates for the decay of maximum tidal shear stresses in the upper flat, and finally tends to make it uniform. This feature is postulated by Friedrichs and Aubrey (1996) among others to get sedimentological equilibrium. Then studying the case of non-rectilinear shore, they concluded that the profile convexity was enhanced within an embayed shoreline, and reduced when the latter is lobate.

Conversely, sediment features can give some insight into the hydrodynamics characterization of a tidal flat. A comparison between the steep Northern flat in the Seine Estuary and the Brouage or Skeffling mudflats, whose slopes are gentle, shows that maximum velocities are similar (around $0.4 \mathrm{~m} \mathrm{~s}^{-1}$, Fig. 5), except in the lower part of the Seine flat where they are larger. These larger velocities $\left(1 \mathrm{~m} \mathrm{~s}^{-1}\right.$ or so) correspond to the sandy area of the flat, whereas the rest is mainly muddy, as the other investigated flats. This confirms a relationship between the nature of sediment and the maximum tidal velocity, as postulated by many authors. As far as cohesive sediment is concerned, this relationship should be based on the ratio between the induced maximum shear stress and the critical shear stress for erosion. Then, assuming (i) fine sediment is available from offshore or from a river input and (ii) the flat is not far from equilibrium, a maximum velocity can be estimated from the sediment nature. By using Eq. (1) for cross-shore currents $(u \sim$ tidal range/slope) and the dependence of longshore current on the square root of local depth, the slope of the flat can be iteratively deduced from the tidal range and the long-shore current off the flat. Such an adaptability of the slope seems to be confirmed by recent morphological studies (e.g. Friedrichs and Aubrey, 1996; Lee and Mehta, 1997; Roberts et al., 2000). In other words, the combined information of topography, long-shore current at the boundary, tidal range, tidal velocities and sediment nature seems redundant. This result, deduced without accounting for wave effects, can be used to describe a lack of balance possibly due to wave erosion - and thus predict a morphological trend. Also this kind of redundance should be kept in mind when aiming at classification of intertidal flats. 


\subsection{Wave effects}

Wave-induced stresses are large on tidal flats, so that erosion is likely to occur. Small incident waves have maximum effect when the local depth equals the "saturation" wave height, that is at the beginning of local flood or at the end of ebb: then sediment resuspended on flood is being transported onto the upper flat where it can deposit during slack (high) water, leading to a possible net accretion. On the other hand, high incident waves - which are often enhanced at high water when the fetch is extended - have maximum effect at high water: in this case resuspended material may be transported offshore by ebb currents and a net erosion will result. These features can change drastically depending on the direction of winds that occur simultaneously. For instance at the Skeffling mudflat, wind velocities over than $5 \mathrm{~m} \mathrm{~s}^{-1}$ have been proved to reverse the shorewards transport of mud (Christie et al., 1999). Nevertheless, effects of strong waves amount to global erosion and then generate concave bottom profiles, a shape that is consistent with uniform wave-induced bottom stress (e.g. Friedrichs and Aubrey, 1996).

In most areas, both tidal flow and wave forcings coexist. The question of their relative importance is often addressed. It can be assessed by bottom stresses computation, based on full two-dimensional mathematical modelling, which is time-consuming especially for waves, or estimated from the simple Eqs. (1) and (6), provided continuous measurements of waves in the middle of the flat are available. As for the sediment behaviour, it has been seen that tidal processes generally generate on shore sediment transport and induce accretionary convex profiles, whereas waves generate erosional concave profiles. We think that any residual equilibrium is somehow virtual and is actually a dynamical one, any flat experiencing periods of tidal accretion and wave erosion, often according to a seasonal cycle (e.g. Kirby et al., 1993). This frequent competition between tidal currents and waves explains why observed tidal flat profiles are not consistent with uniformity of maximum velocity or shear stress or dissipation rate. Effectively, neither computations nor measurements conducted on the three investigated sites showed any uniformity across the flats.

\section{Conclusion}

Hydrodynamics of intertidal flats result from the combination of several forcings, mainly the tidal currents, the waves and associated winds, and drainage.

Tidal currents can be split into cross-shore and long-shore components. The former is due to filling and emptying of the embayment over the flat. If the bottom slope is regular, the maximum value of this cross-shore flow is uniform and inversely proportional to the slope in the lower flat, and decreases in the upper flat. The long-shore component can develop when the flat is connected to an estuary and is not bounded by headlands; across the flat, this long-shore component is roughly proportional to the square root of the water depth.

Wave measurements on gently sloping flats indicate that wave heights are limited by a fraction of the water depth, whatever be the incident wave height, because of 
wave energy dissipation; the importance of this effect is quantified by the nondimensional parameter $f_{\mathrm{w}} / \beta$ (ratio between the wave friction factor and the bottom slope). This local saturation of wave height can be of practical use for modelling, as it gives an idea of the maximum wave height related to a given forcing, without running a costly wave propagation model. In addition, the maximum-induced bottom stress is proved to occur at high water and to be proportional to the maximum water depth.

Combined with mathematical modelling, observations on the three investigated sites provided validations of these schematic considerations. Besides, despite different forcing ranges and topographic configurations, maximum velocities have been proved similar on muddy bottoms, indicating some relationship between the sediment nature and tidal velocities.

Consequences of tide and wave forcings on sediment transport have been listed. Schematically, tidal asymmetry induces onshore sediment transport, then generates accretion on the upper flat leading to a convex bottom profile, which in turn favours an ebb dominance that enhances the seawards sediment transport etc. Finally a tidal equilibrium is likely to occur, with a resulting slope and convexity. Such an equilibrium can be upset by wavy episodes that erode the flat, prevent deposition on the upper flat and favour offshore transport.

Among the other hydrodynamical forcings, drainage is an important process, very specific to tidal flats. Even if the induced shear stresses are not very high, drainage contributes to the offshore transport of sediment that have been previously resuspended and freshly deposited on high water slack. Combined with tidal currents, drainage is proposed to be responsible for the formation of longitudinal bedforms, which often constitute a network of parallel ridges and runnels located where the slope is minimum.

\section{Acknowledgements}

The authors thank R. Silva Jacinto (IFREMER) for helpful discussions on the wave effects, H. Jestin (IFREMER) for contributing to field experiments, D. Gouleau, J. Germaneau, P-G Sauriau and S. Robert (CREMA-La Rochelle) for sharing their knowledge on the Brouage mudflat and W. Van Der Lee (IMAU, Utrecht Univ.) for complementary data on the Skeffling mudflat. They also thank K. Dyer and other reviewers for valuable comments on the manuscript. The study has been partly supported by the INTRMUD project, which is funded by the Commission of the European Union, Directorate General for Science, Research and Development, under contract $\mathrm{N}^{\circ}$ MAS3-CT95-0022.

\section{Appendix A. Maximum tidal current on a flat with uniform bottom slope}

Assuming that the water elevation remains horizontal whatever be the tidal phase, the magnitude of the cross-shore current can be estimated by considering the conservation of volume as the water level rises and falls (Friedrichs and Aubrey, 1996). If the 
water level rises, the edge of the wetted area moves landward by a distance determined by the slope of the mudflat. The volume of additional water that must pass through a plane (at location $x$ ) parallel to the shore depends on the rise in water level multiplied by the distance from the plane to the water's edge.

Friedrichs and Aubrey (1996) show that the cross-shore current speed is given by

$$
u(x, t)=\frac{x_{f}(t)-x}{h(x, t)} \frac{\mathrm{d} z}{\mathrm{~d} t}
$$

where $x_{\mathrm{f}}(t)$ is the location of the water's edge at time $t$, referred to as the tidal front by Friedrichs and Aubrey (1996) and others. If the slope of the mudflat is approximately uniform, the first term on the right-hand side of Eq. (1) is equal to the inverse of the mudflat slope $\beta$.

Assuming a sinusoidal variation of the water level $z$, its rate of change can be derived as

$$
\frac{\mathrm{d} z}{\mathrm{~d} t}=\frac{\pi R}{T_{\text {tide }}} \cos \left(\frac{2 \pi t}{T_{\mathrm{tide}}}\right)
$$

where $R$ is the tidal range and $T_{\text {tide }}$ is the tidal period. Substituting for the rate of change of water level from Eq. (2), Eq. (1) becomes

$$
u=\frac{1}{\beta} \frac{\pi R}{T_{\text {tide }}} \cos \left(\frac{2 \pi t}{T_{\text {tide }}}\right) \text { for } x<x_{\mathrm{f}}(t) .
$$

Thus for every location of the flat which is covered when the rate of water level variation is maximum (i.e. the section of the flat below the mean water level), the maximum cross-shore current is approximately [cosine $=1$ in (A.3)]:

$$
u_{\max }=\frac{\pi R}{\beta T_{\text {tide }}} .
$$

In the upper part of the flat (above mean sea level), Friedrichs and Aubrey (1996) show that the maximum current occurs at the tidal front, and the intensity is given by

$$
u_{\max }=\frac{\mathrm{d} x_{\mathrm{f}}}{\mathrm{d} t}=\frac{1}{\beta} \frac{\mathrm{d} z}{\mathrm{~d} t}=\frac{\pi R}{\beta T_{\text {tide }}} \cos \left(\arcsin \left(\frac{2 \beta}{R} x_{f}\right)\right)=\frac{\pi R}{\beta T_{\text {tide }}} \sqrt{1-\left(\frac{2 \beta}{R} x_{\mathrm{f}}\right)^{2}},
$$

with $x_{\mathrm{f}}=0$ at mid-tide.

\section{Appendix B. Wave height distribution on a dissipative flat with uniform bottom slope}

Let us consider the cross-shore conservation of wave energy in steady state, without wave refraction, nor generation

$$
\frac{\mathrm{d}}{\mathrm{d} x}\left(E(x) C_{\mathrm{g}}(x)\right)=-D(x)
$$


where $C_{\mathrm{g}}$ is the wave group velocity, $D$ the dissipation by bottom friction and $E$ the total wave energy, that can be evaluated as

$$
E=\frac{1}{8} \rho g H^{2},
$$

where $\rho$ is the water density and $g$ the acceleration due to gravity. In the case of a monochromatic wave, $D$ equals the work of a quadratic bottom friction at the speed of the orbital velocity during one wave period, that is

$$
D=\frac{1}{T} \int\left(\frac{\rho}{2} f_{\mathrm{w}} u_{\mathrm{w}}\left|u_{\mathrm{w}}\right|\right) u_{\mathrm{w}} \mathrm{d} t
$$

where $T$ is the wave period, $u_{\mathrm{w}}$ the orbital velocity above the boundary layer and $f_{\mathrm{w}}$ a wave friction factor. The term between parentheses represents the wave-induced shear stress. In the case of a sinusoidal wave with a maximum velocity $U_{\mathrm{w}}, D$ can be integrated

$$
D=\frac{2}{3 \pi} \rho f_{\mathrm{w}} U_{\mathrm{w}}^{3} .
$$

In shallow waters, $C_{\mathrm{g}}=(g h)^{1 / 2}$, and $U_{\mathrm{w}}$ can be approximated by

$$
U_{\mathrm{w}}=\frac{H}{2} \sqrt{\frac{g}{h}} .
$$

Eq. (5) is valid for $h \ll\left(3 g / 4 \pi^{2}\right) T^{2} \cong 0.75 T^{2}$.

Finally, the energy conservation reduces to

$$
\frac{\mathrm{d}}{\mathrm{d} x}\left(H^{2} h^{1 / 2}\right)=-\frac{2 f_{\mathrm{w}}}{3 \pi} H^{3} h^{-3 / 2} .
$$

In the case of a uniform slope, $h=\beta x$, with $x=0$ at the shore but $>0$ seaward, then Eq. (6) becomes

$$
\frac{\mathrm{d}}{\mathrm{d} x}\left(H^{2} x^{1 / 2}\right)=\frac{2}{3 \pi} \frac{f_{\mathrm{w}}}{\beta^{2}} H^{3} x^{-3 / 2}
$$

\section{References}

Anderson, F.E., 1981. The Northern muddy intertidal: seasonal factors controlling erosion and deposition - A review. Canadian Journal of Fisheries and Aquatic Science 40 (Suppl. 1), 143-159.

Anderson, F.E., Howell, B.A., 1984. Dewatering of an unvegetated muddy tidal flat during exposure: dessication or drainage? Estuaries 7-3, 225-232.

Bassoullet, Ph., Le Hir, P., Gouleau, D., Robert, S., 2000. Sediment transport on an intertidal mudflat: field investigations and estimation of fluxes within the Baie de Marennes-Oléron (France). 20 (12/13), 1635-1653.

Brenon, I., Le Hir, P., 1999. Modelling the turbidity maximum in the Seine estuary (France): identification of formation processes. Estuarine, Coastal and Shelf Science 49, 525-544.

Christie, M.C., Dyer, K.R., Turner, P., 1999. Sediment flux and bed level measurements from a macrotidal mudflat. Estuarine, Coastal and Shelf Science 49, 667-688. 
Collins, M.B., Ke, X., Gao, S., 1998. Tidally-induced flow structure over intertidal flats. Estuarine, Coastal and Shelf Science 46, 233-250.

Dronkers, J., 1986. Tidal asymmetry and estuarine morphology. Netherlands Journal of Sea Research 20 $(2 / 3), 117-131$.

Dyer, K.R., Christie, M.C., Wright, E.W., 2000. The classification of intertidal mudflats 20 (10/11), $1037-1038$.

Eisma, D., 1997. Intertidal Deposits: River Mouths, Tidal Flats and Coastal Lagoons. Marine Science Series, CRC Press, Boca Raton, 507 p.

Friedrichs, C.T., Aubrey, D.G., 1996. Uniform bottom shear stress and equilibrium hypsometry of intertidal flats. In: Pattiaratchi, C. (Ed.), Mixing in Estuaries and Coastal Seas, Coastal and Estuarine Studies, vol. 50. American Geophysical Union, Washington DC, pp. 405-429.

Friedrichs, C.T., Madsen, O.S., 1992. Nonlinear diffusion of the tidal signal in frictionally dominated embayments. Journal of Geophysical Research 97, $\mathrm{N}^{\circ} \mathrm{C} 4,5637-5650$.

Guarini, J.M., Blanchard, G.F., Gros, Ph., Harrison, S.J., 1997. Modelling the mud surface temperature on intertidal flats to investigate the spatio-temporal dynamics of the benthic microalgal photosynthetic capacity. Marine Ecology progress Series 153, 25-36.

Kirby, R., Bleakley, R.J., Weatherup, S.T.C., Raven, P.J., Donaldson, N.D., 1993. Effect of episodic events on tidal mud flat stability, Ardmillan Bay, Strangford Lough, Northern ireland. In: Mehta, A.J. (Ed.), Nearshore and Estuarine Cohesive Sediment Transport, Coastal and Estuarine Studies No 42, AGU, pp. 378-392.

Lee, S.C., Mehta, A.J., 1997. Problems in characterizing dynamics of mud shore profiles. ASCE Journal of Hydraulic Engineering 123 (4), 351-361.

Li, Y., Mehta, A.J., 1997. In: Burt, N., Parker, R., Watts, J. (Eds.), Mud fluidization by water waves. Cohesive Sediments. Wiley, New York, pp. 341-352.

Pethick, J.S., 1980. Velocity surges and asymmetry in tidal channels. Estuarine and Coastal Marine Science 11, 331-345.

Postma, H., 1961. Transport and accumulation of suspended matter in the Dutch Wadden Sea. Netherland Journal of Sea Research 1, 148-190.

Roberts, W., Le Hir, P., Whitehouse, R.J.S., 2000. Investigation using simple mathematical models of the effect of tidal currents and waves on the profile shape of intertidal mudflats $20(10 / 11), 1079-1097$.

Roberts, W., Whitehouse, R.J.S., 2000. Predicting the profile of intertidal mudflats formed by cross-shore tidal currents. Proceedings of INTERCOH'98, Korea, in press.

Silva Jacinto R., Bessineton, Ch., Levoy, F., Védieu, C.H., Lesourd, S., Rousset, H., Benoît, L., Jestin, H., Monfort, O., 1998. Réponse de la vasière Nord aux forçages météo-océaniques. Rapport final du thème Hydrodynamique et Transport Sédimentaire, programme scientifique Seine Aval, avril 1998, pp. 99-111.

Soulsby, R.L., Hamm, L., Klopman, G., Myrhaug, D., Simons, R.R., Thomas, G.P., 1993. Wave-current interaction within and outside the bottom boundary layer. Coastal Engineering 21, 41-69.

Uncles, R.J., 1981. A note on tidal asymmetry in the severn estuary. Estuarine, Coastal and Shelf Science 13, 419-432.

Wells, J.T., Kemp, G.P., 1986. Interaction of surface waves and cohesive sediments: field observations and geologic significance. In: Mehta, A.J. (Ed.), Estuarine Cohesive Sediment Dynamics, Lecture notes on Coastal and Estuarine Studies $N^{\circ} 14$. Springer, Berlin, pp. 43-65.

Whitehouse, R.J.S, Bassoullet, P., Dyer, K.R., Mitchener, H.J., Roberts, W., 2000. The influence of bedforms on flow and sediment transport over intertidal mudflats. Continental Shelf Research.

Whitehouse, R.J.S., Mitchener, H.J., 1998. Observations of the morphodynamic behaviour of an intertidal mudflat at different timescales. In: Black, K.S., Paterson, D.M., Cramp, A. (eds) Sedimentary Processes in the Intertidal Zone. Geological Society, London, Special Publication 139, pp. 255-271. 